\title{
ELECTRIC FIELD INDUCED DOMAIN FORMATION IN SURFACE STABILIZED FERROELECTRIC LIQUID CRYSTAL CELLS
}

\author{
I. Dierking, F. Gießelmann, J. Schacht and P. Zugenmaier
}

Institut für Physikalische Chemie, TU Clausthal, Arnold-Sommerfeld-Str. 4, D-38678 Clausthal-Zellerfeld, Germany

Keywords: Liquid Crystals, Ferroelectricity, Chirality, Smectic C*, Mesophse

\begin{abstract}
Two types of domains have been observed for $\mathrm{S}_{\mathrm{C}}{ }^{*}$ ferroelectric liquid crystals in surface stabilized cells (SSFLC) by application of a high electric field with the smectic layers tilted by the amount of the chevron angle with respect to the normal of the rubbing direction in the substrate plane. The layer structure resembles that of a chevron configuration in the plane of the substrate similar to the recently reported stripe-shaped SSFLC structure. The two domain types "appear" to switch in a reciprocal fashion when applying an AC electric field and observed between crossed polarizers. The temperature dependence of this effect has been investigated and an explanation proposed analogous to a striped texture model.
\end{abstract}




\section{INTRODUCTION}

A variety of director configurations can be observed in $\mathrm{S}_{\mathrm{C}}^{*}$ ferroelectric liquid crystals, depending on anchoring strength and geometry of the sample. In thick samples prepared on a glass slide without orientation layers, typical textures may appear, e.g. the well known fan-shaped texture. The fans may be modulated with equidistant disclination lines due to a helical director configuration [1,2]. This line texture may also be observed in LC cells with a small gap and orientation layers depending on the value of the $\mathrm{S}_{\mathrm{C}}^{*}$ pitch [3]. If the cell gap is comparable to or smaller than the $S_{C}^{*}$ pitch, the helix is usually supressed by surface effects and a so called surface stabilized ferroelectric liquid crystal (SSFLC) geometry is obtained [3]. In the simplest case, one observes a structure, where the layers are arranged perpendicular to the substrate plane and to the rubbing direction of the orientational layers with the molecules tilted with respect to the layer normal (bookshelf geometry). Due to anchoring effects at the substrate, one usually observes a structure with the layers tilted with respect to the substrate normal (chevron geometry $[4,5])$.

Recently an electric field induced layer structure has been observed, which resembles that of a chevron turned by $90^{\circ}$. The smectic layers are tilted with respect to the normal of the rubbing direction in the substrate plane by the amount of the chevron angle ( $\Delta$ to the director tilt angle), in contrast to the well known chevron geometry, where the smectic layers are tilted with respect to the substrate normal. Application of a high electric field to the LC cell results in the formation of two domain types, which seem to switch in an reciprocal way when applying an AC electric field and placed between crossed polarizers. The formation of the two domain types is analogous to observations by PATEL et al. [6,7], although the conditions applied are quite different. The measurements were carried out on a diarylethane- $\alpha$-chloroester,

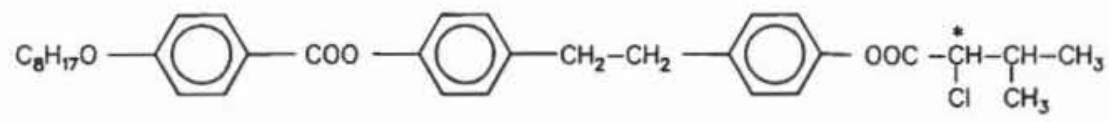

derived from D-valine, which will be referred to as compound D8. Synthesis, purification, characterization and temperature dependent measurements of several physical parameters of this compound and other homologous of the series will be published elsewhere [8].

A detailed review on textures of liquid crystalline phases can be found in a book by DEMUS/RICHTER [9] and GRAY/GOODBY [10]. A recent review on properties of liquid crystals was published by CHANDRASEKHAR [11]. Properties and applications of ferroelectric liquid crystals are summarized in a book by GoodBY et al. [12]. 


\section{EXPERIMENTAL}

The phase sequence of D8 as studied by polarization microscopy (Olympus BH-2, equipped with a Mettler FP 52 hot stage and a Mettler FP 5 temperature controller) and DSC measurements (Perkin Elmer DSC 7) is given by:

$$
\text { I } 134.5 \mathrm{~N}^{*} 129.4 \text { TGB A}^{*} 129.2 \mathrm{~S}_{\mathrm{A}}^{*} 125.6 \mathrm{~S}_{\mathrm{C}}^{*} 92.9 \mathrm{~S}_{\mathrm{I}}^{*} 83.7 \mathrm{~S}_{\mathrm{F}}^{*}
$$

All measurements presented below were performed on samples prepared in commercially available liquid crystal cells (E.H.C. Co. Ltd.) with a cell gap of $4 \mu \mathrm{m}$, ITO electrodes and parallel rubbed polyimide orientation layers.

Determination of the value of the optical director tilt angle and the angular position of the layer normal was performed using a method analogous to the one introduced by BAHR et al.[13]. We applied an electric square wave field with frequency $\mathrm{f}=200 \mathrm{~Hz}$ and an amplitude of $\mathrm{E}=1 \mathrm{MV} / \mathrm{m}$. The cell is placed in the hot stage with the rubbing direction $\vec{x}$ coinciding with an angular position of the hot stage marking $\varphi=0^{\circ}$. Recording the transmissions of the two switching states with "polarization up" $\left(\mathrm{I}_{\text {up }}\right)$ and "polarization down" $\left(\mathrm{I}_{\text {down }}\right)$ as a function of the rotation angle $\varphi$, which is defined as the rotation angle of the hot stage in the substrate plane, one can determine the director tilt angle $\theta$ by the phase shift between the $\mathrm{I}_{\mathrm{up}}(\varphi)$ and $\mathrm{I}_{\text {down }}(\varphi)$ curves and the angular position of the layer normal $\overrightarrow{\mathrm{k}}$ with respect to the rubbing direction $\vec{x}$ from the intersection of the $I_{\text {up }}(\varphi)$ and $I_{\text {down }}(\varphi)$ curves.

\section{EXPERIMENTAL RESULTS}

The first series of measurements was obtained by slowly heating the oriented sample in SSFLC cells through the $S_{C}^{*}$ phase and recording the $I_{\text {up }}(\varphi)$ and $I_{\text {down }}(\varphi)$ data for several different temperatures. These data are depicted in figure 1 for temperatures $T=118,120$, $122,123,124$ and $125^{\circ} \mathrm{C}$.

It is clear, that the phase shift between two respective curves (i.e. the angular difference between the two minima of intensity) and, therefore the director tilt angle $\theta$ decreases with increasing temperature, as expected. The intersection points of the two corresponding curves remain constant at an rotation angle $\varphi=0^{\circ}$, which means that the position of the layer normal $\vec{k}$ is independent of temperature and encloses an angle $\alpha=0^{\circ}$ with the rubbing direction $\vec{x}$, i.e. rubbing direction and layer normal coincide for all temperatures. The structure of this configuration is the well known geometry depicted in figure 2(a)-(c) as a top view of the cell. At zero electric field, two spontaneously formed domains are observed, one in the polarization "up" and one in the polarization "down" state (figure 2(a)). The angle between the respective directors $\vec{n}$ is given by $2 \theta$. Applying an electric field $E$ 


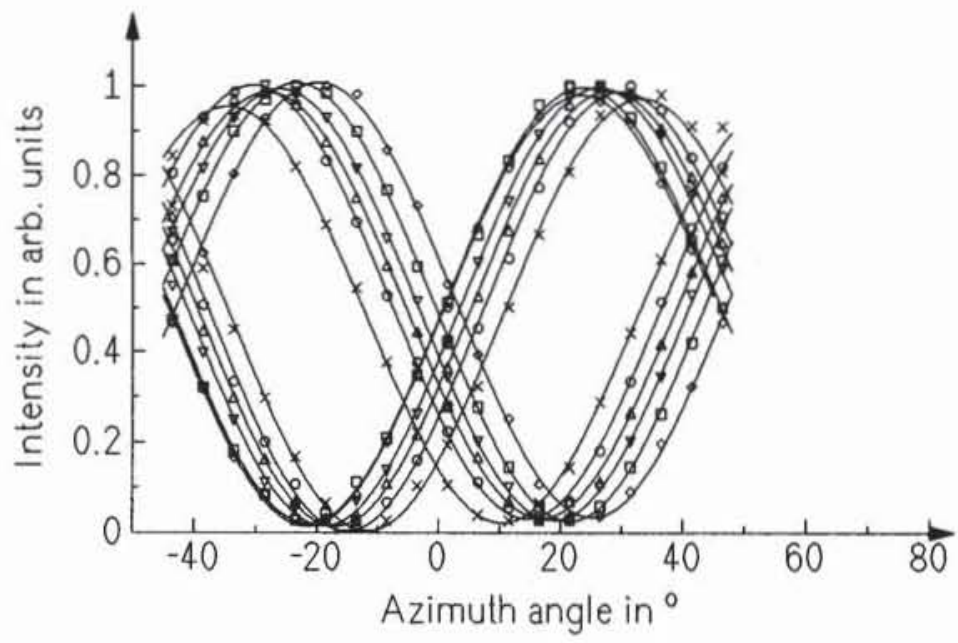

Figure 1: Intensity of the transmitted light as a function of the rotation angle. $(\diamond)$ $\mathrm{T}=118^{\circ} \mathrm{C}$, (ㅁ) $\mathrm{T}=120^{\circ} \mathrm{C}$, ( $\left.\nabla\right) \mathrm{T}=122^{\circ} \mathrm{C},(\Delta) \mathrm{T}=123^{\circ} \mathrm{C}$, (O) $\mathrm{T}=124^{\circ} \mathrm{C}$ and $(X)$ $\mathrm{T}=125^{\circ} \mathrm{C}$.

perpendicular to the substrate plane in the $-\vec{z}$ direction, all molecules reorient in the polarization "down" state and the director $\overrightarrow{\mathrm{n}}$ encloses an angle $\theta$ with the layer normal $\overrightarrow{\mathrm{k}}$ (figure 2(b)). Reversing the direction of the electric field, the molecules reorient by an angle $2 \theta$ into the polarization "up" state (figure 2(c)).

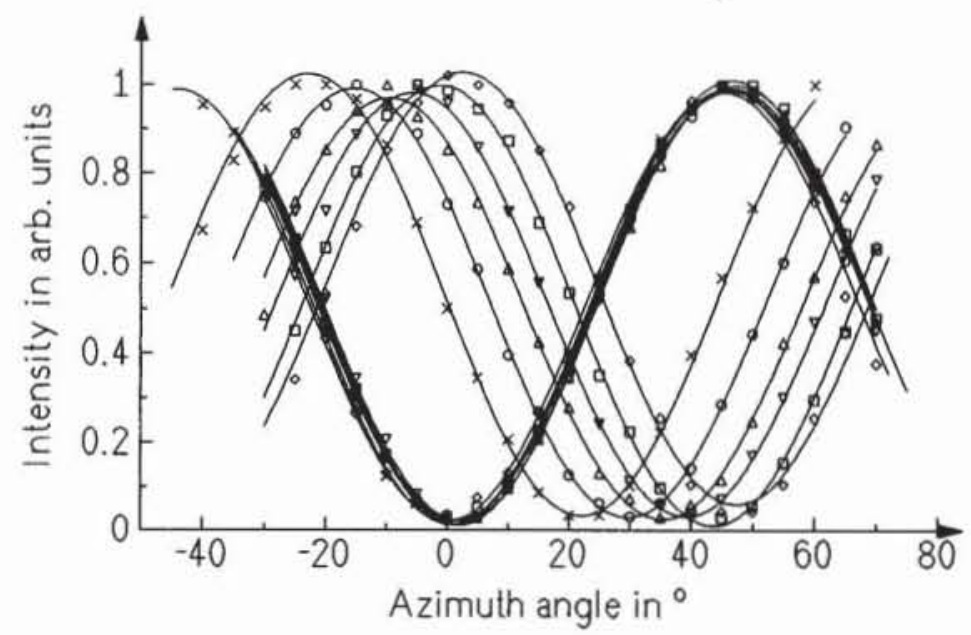

Figure 3: Second measurement series after the high field treatment $(\diamond) \mathrm{T}=118^{\circ} \mathrm{C}$, (口) $\mathrm{T}=120^{\circ} \mathrm{C},(\nabla) \mathrm{T}=122^{\circ} \mathrm{C},(\Delta) \mathrm{T}=123^{\circ} \mathrm{C},(\mathrm{O}) \mathrm{T}=124^{\circ} \mathrm{C}$ and $(X) \mathrm{T}=125^{\circ} \mathrm{C}$. 

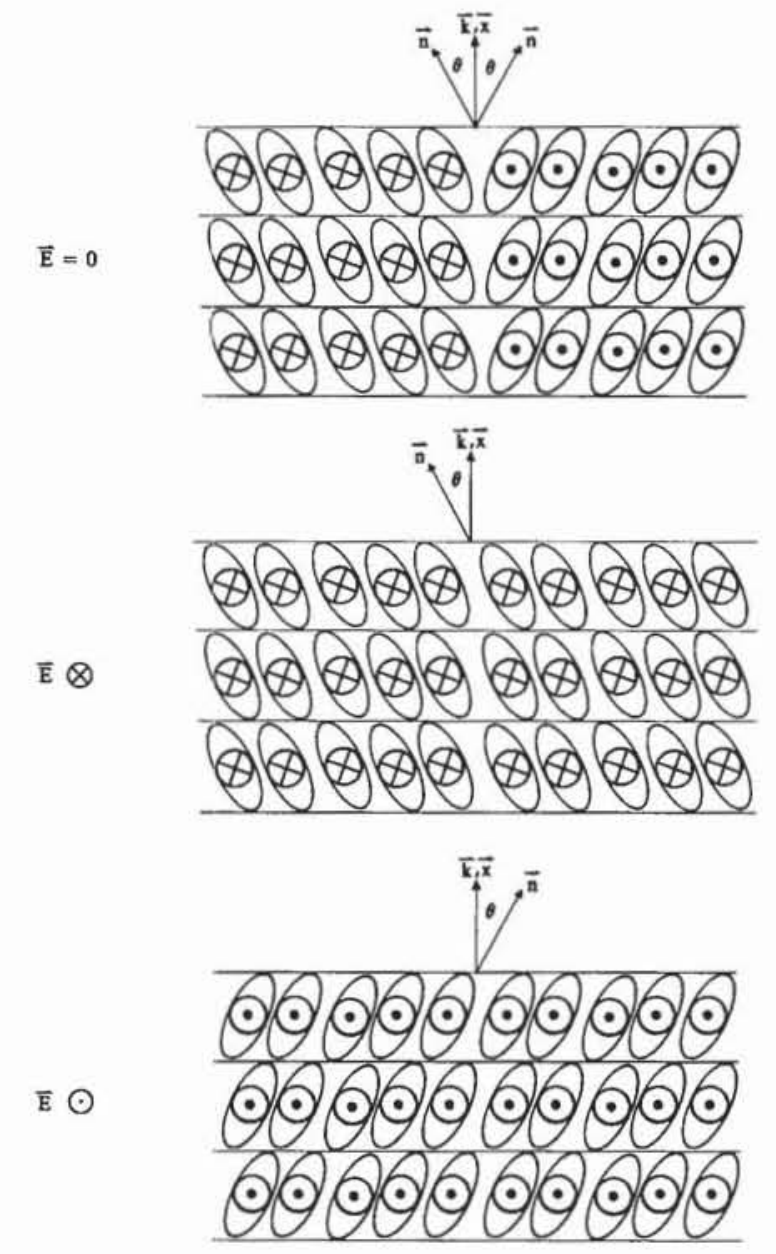

Figure 2: Top view of the SSFLC cell for different directions of the electric field $E$, before the high field treatment. (a) $E=0$, (b) $E$ in $-z$ direction and (c) $E$ in $+z$ direction.

The second series of measurements was obtained after application of a high electric field with amplitude $\mathrm{E}=10 \mathrm{MV} / \mathrm{m}$ at $\mathrm{T}=115^{\circ} \mathrm{C}$ for about 5 seconds. The $\mathrm{I}_{\text {up }}(\varphi)$ and $\mathrm{I}_{\text {down }}(\varphi)$ curves are now considerably different from the respective ones of the first series and are depicted in figure 3 . The phase shift between two respective curves is still the same as before, but the angular position of the layer normal has changed and is now dependent on temperature. The director tilt angle still has the same value as in the first measurement series (figure 4), but the angle $\alpha$ between the rubbing direction $\vec{x}\left(\varphi=0^{\circ}\right)$ and the layer normal $\vec{k}$ is now equal to the director tilt angle $(\alpha=\theta)$. Figure 5 depicts the dependence of the layer 
angle $\alpha$ on the director tilt angle $\theta$ (parameter temperature). The line shows a least-square fit, which yields a slope of 1.007 , leading to $\alpha(\mathrm{T})=\theta(\mathrm{T})$, disregarding the small offset angle $\alpha=0.7^{\circ}$ for $\theta=0^{\circ}$.

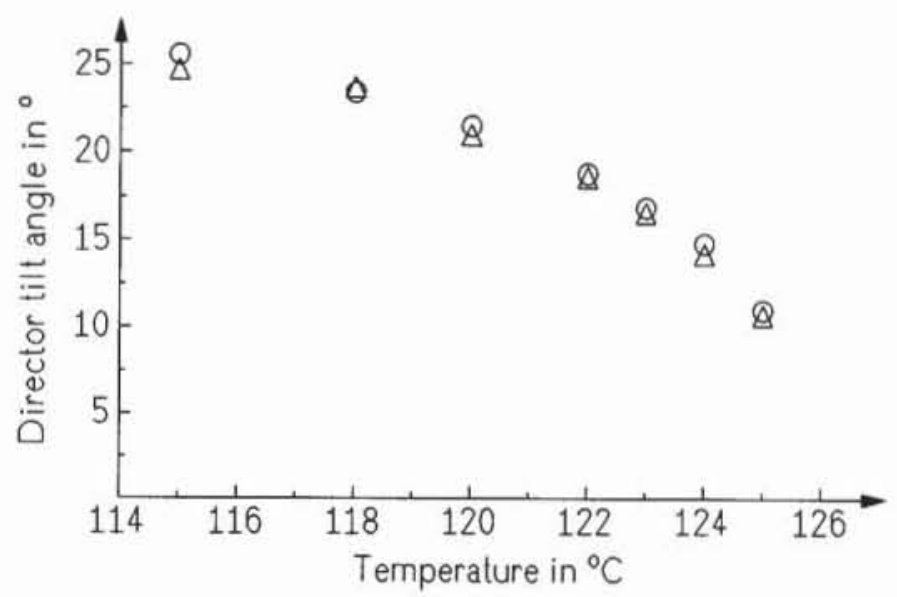

Figure 4: Director tilt angle $\theta$ as a function of temperature: $(O)$ first measurement series and $(\Delta)$ second measurement series.

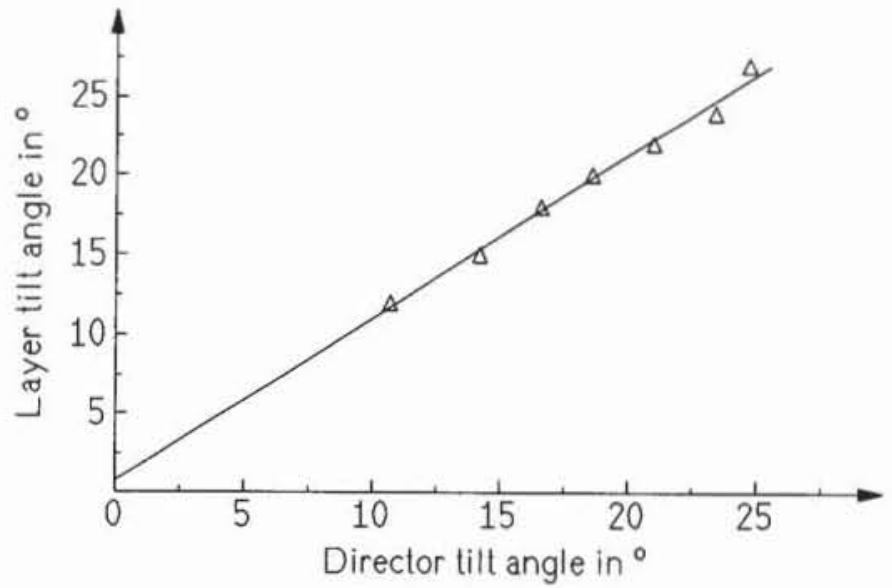

Figure 5: Layer tilt angle $\alpha$ as a function of the director tilt angle $\theta$. 
A structural model which explaines this behaviour is depicted in figure 6(a)-(c) and supported by texture photographs (figure $7(\mathrm{a})-(\mathrm{c})$ ) taken at a hot stage position $\varphi=0^{\circ}$ and temperature $\mathrm{T}=110^{\circ} \mathrm{C}$. After the high field treatment the molecules are oriented, with the
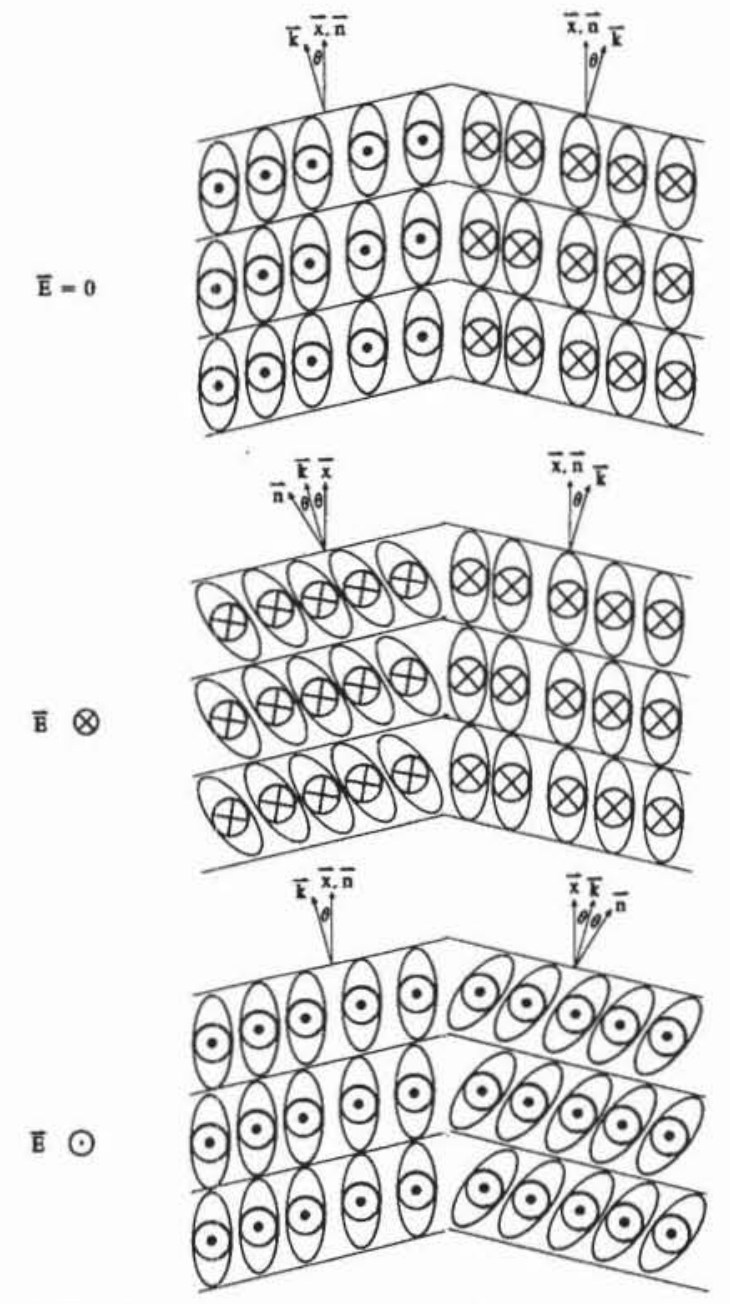

Figure 6: Top view of the cell after the high field treatment (a) $E=0$, (b) $E$ in $-z$ direction and (c) $\mathrm{E}$ in $+\mathrm{z}$ direction.

director $\overrightarrow{\mathrm{n}}$ coinciding with the rubbing direction $\overrightarrow{\mathrm{x}}$. This is only possible, if the layers reorient by an angle $\alpha=\theta$, since the director tilt angle is essentially fixed for a given temperature. At zero field after the high field treatment, the two domain types " 1 " and "2", newly formed, have the same director orientation $\bar{n}$ (figure 6(a)) and are in the dark state between crossed polarizers (figure 7(a)). Applying an electric field perpendicular to the 
substrate plane in the $-\vec{z}$ direction, molecules of domain type "1", which are in the polarization "up" state, switch by an angle $2 \theta$ along the tilt cone, while the molecules of domain type " 2 ", which are already in the polarization "down" state, stay in their position (figure 6(b) and figure 7(b)). Reversing the direction of the electric field, all molecules reorient along the tilt cone by an

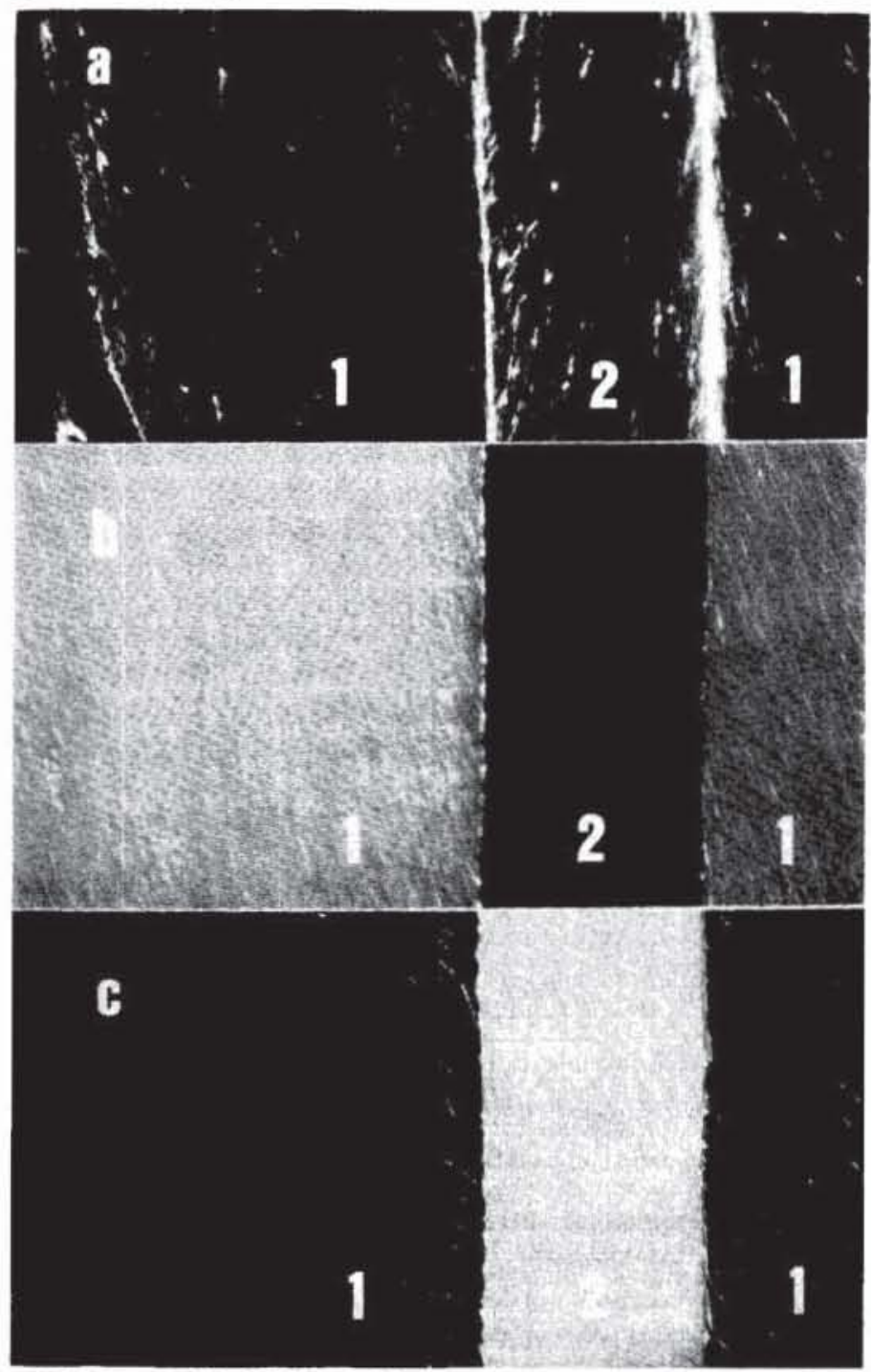

Figure 7: Textures of the field induced domains between crossed polarizers: (a) $E=0 \mathrm{~V}$, (b) $\mathrm{E}=-2 \mathrm{MV} / \mathrm{m} \mathrm{DC}$ and (c) $\mathrm{E}=+2 \mathrm{MV} / \mathrm{m} \mathrm{DC}$. The depicted area is $1 \times 0.5 \mathrm{~mm}^{2}$ in each case. 
angle $2 \theta$ and domain type " 1 " now optically appears like domain type "2" before, and vice versa (figure $6(\mathrm{c})$ and figure $7(\mathrm{c})$ ). Observing the cell between crossed polarizers while applying a low frequency square wave field, the switching between the configurations depicted in figure 6(b) and 6(c) optically "appears" as if the two domain types switch in a reciprocal fashion (figures 7 (b) and $7(\mathrm{c})$ ) due to the different layer orientations in both domain types.

\section{DISCUSSION}

Recently, several papers have discussed models for the stripe-shaped texture [14-18]. The field induced domain formation in SSFLC cells can be explained by a model proposed by SHAO et al.[14] for the stripe-shaped texture. It was usually found, that the width of the stripes is approximately equal to the cell gap. The formation of large field induced domains as observed in our case, which may extend across the whole electrode area of the cell, can be described by an analogous mechanism as proposed in [14].

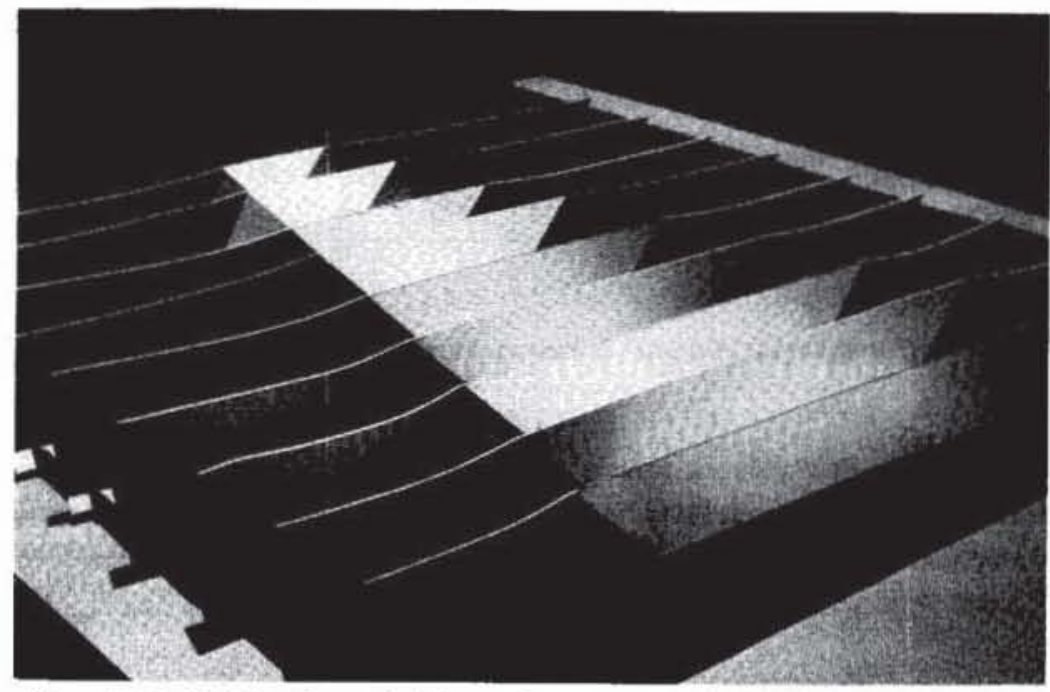

Figure 8 (a): Model of the vertical chevron layer structure, the initial structure in SSFLC cells before the high field treatment.

The initial layer structure in the cell before the high field treatment is of the vertical chevron type $[4,5]$ with zig-zag defects mediating regions of chevron shaped layers, tilted in opposite direction [5]. These intermediate zones are of bookshelf type with layers tilted by the amount of the chevron angle with respect to the rubbing direction [14]. This structure is depicted in the model of figure 8(a). The high field treatment forces the chevron layers to 
straighten up as has been demonstrated in several publications [19-21]. This means that by application of a reasonably high electric field, the bookshelf areas, previously mediating two regions of opposite chevron direction, grow at the expense of the chevron regions, until the layer structure in the cell is of bookshelf type. In this configuration the layer normal is inclined to the rubbing direction by the amount of the previous chevron angle. The intermediate zone between two bookshelf regions of opposite tilt is of the chevron type. The layer structure of the cell now resembles that of a chevron structure turned by $90^{\circ}$ resulting in a horizontal chevron structure which is depicted in the model of figure $8(b)$.

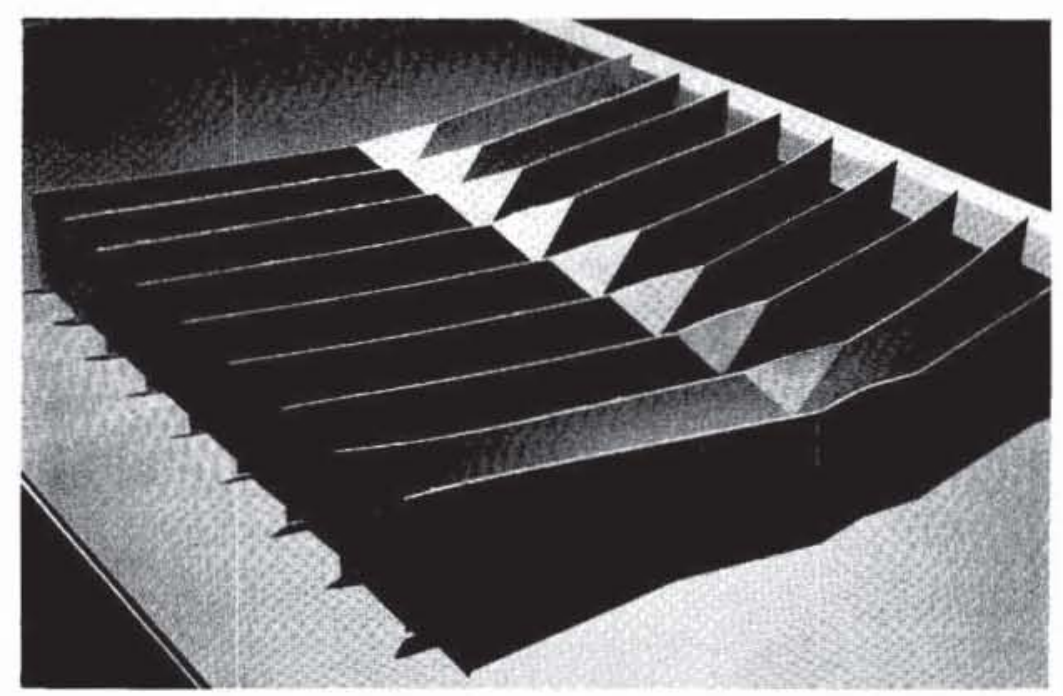

Figure 8 (b): Model of the horizontal chevron structure after the high field treatment.

The here investigated field induced domain formation in SSFLC cells is the transition from a vertical chevron structure with bookshelf intermediate zones between regions of opposite chevron direction to a horizontal chevron structure with chevron intermediate zones between bookshelf areas with opposite tilt of the layer normal with respect to the rubbing direction.

\section{CONCLUSIONS}

The electric field induced domain formation in SSFLC cells has been investigated by polarization microscopy and electrooptical methods. The layer structure of the cell after the high field treatment resembles that of a horizontal chevron structure with the layer normal tilted with respect to the rubbing direction by the amount of the previous chevron angle, which in our case is equal to the director tilt angle. The process of changing the initial 
vertical chevron structure to the domain structure can be described in terms of a model [14] proposed for the stripe-shaped texture, although there is no relation between the size or shape of the domains and the cell gap, as it is found for stripe-shaped textures.

This work was supported by a grant from the Deutsche Forschngsgemeinschaft.

\section{REFERENCES}

[1] Martinot-Lagarde, PH., 1976, Jour. de Phys., 37, C3-129

[2] Martinot-Lagarde, Ph., Duke, R. and Durand, G., 1981, Mol. Cryst. Liq. Cryst., 75, 249

[3] Clark, N.A. and Lagerwall, S.T., 1980, Appl. Phys. Lett., 36, 899

[4] Rieker, T.P., Clark, N.A., Smith, G.S., Parmar, D.S., Sirota, E.B. and SAFINYA, C.R., 1987, Phys. Rev. Lett., 59, 2658

[5] CLARK, N.A. and RIEKER, T.P., 1988, Phys. Rev. A, 37, 1053

[6] Patel, J.S. and GoodBy, J.W., 1986, J. Appl. Phys., 59, 2355

[7] PATEL, J.S., Lee, Sin-DOo and GoodBy, J.W., 1989, Phys. Rev. A, 40, 2854

[8] Dierking, I., Giebelmann, F., Kuberow, J. and Zugenmaier, P., submitted to Liq. Cryst.

[9] Demus, D. and Richter, L., Textures of Liquid Crystals, Verlag Chemie, Weinheim, 1978

[10] GraY, G.W. and GoodBY, J.W., Smectic Liquid Crystals-Textures and Structures, Leonard Hill, Glasgow, 1984

[11] Chandrasekhar, S., Liquid Crystals, 2. Edition, Cambridge University Press, Cambridge, 1992

[12] GoodBy, J.W. et al., Ferroelectric Liquid Crystals-Principles, Properties and Applications, Gordon and Breach Science Publishers, Philadelphia, 1991

[13] BAHR, CH. and HePPKE, G., 1987, Liq. Cryst., 2, 825

[14] Shao, R.F., WILlis, P.C. and Clark, N.A., 1991, Ferroelectrics, 121, 127

[15] LeJCEK, L. and PIRKL, S., 1990, Liq. Cryst., 8, 871

[16] FünfschIlling, J. and SCHADT, M., 1991, Jap. Jour. Appl. Phys., 30, 741

[17] JAKLI, A. and SAUPE, A., 1992, Phys. Rev. A, 45, 5674

[18] AsAo, Y. and UCHIDA, T., 1993, Jap. Jour. Appl. Phys. Lett., 32, L604

[19] Johno, M., Chandani, A.D.L., OUChI, Y., Takezoe, H., Fukuda, A., ICHIHASHI, M. and FURUKAWA, K., 1989, Jap. Jour. Appl. Phys. Lett., 28, L119

[20] ItOH, K., JohNo, M., TAKANISHI, Y., OUCHI, Y., TAKEZOE, H. and FUKUdA, A., 1991, Jap. Jour. Appl. Phys., 30, 735

[21] OH-E, M., Isogai, M. and Kitamura, T., 1992, Liq. Cryst., 11, 101 